\title{
Automatic Image Analysis and Classification for Urinary Bacteria Infection Screening
}

\author{
Paolo Andreini ${ }^{1}$, Simone Bonechi $\left.{ }^{1(}\right)$, Monica Bianchini ${ }^{1}$, \\ Alessandro Mecocci ${ }^{1}$, and Vincenzo Di Massa ${ }^{2}$ \\ ${ }^{1}$ University of Siena - Department of Information Engineering and Mathematics, \\ Roma 56, I-53100 Siena, Italy \\ simo_bone@alice.it \\ http://www.diism.unisi.it \\ 2 Diesse Ricerche S.r.l., C/o TLS via Fiorentina 1, I-53100 Siena, Italy
}

\begin{abstract}
In this paper, we present an automatic system for the screening of urinary tract infections. It is estimated that about 150 million infections of this kind occur world wide yearly, giving rise to roughly five billion health-care expenditures. Currently, Petri plates seeded with infected samples are analyzed by human experts, an error prone and lengthy process. Nevertheless, based on image processing techniques and machine learning tools, the recognition of the bacterium type and the colony count can be automatically carried out. The proposed system captures a digital image of the plate and, after a preprocessing stage to isolate the colonies from the culture ground, accurately identifies the infection type and severity. Moreover, it contributes to the standardization of the analysis process, also avoiding the continuous transition between sterile and external environments, which is typical in the classical laboratory procedure.
\end{abstract}

Keywords: Advanced image processing - Support vector machines · Urinoculture screening

\section{Introduction}

Recent technological advances in biomedical engineering and biomedicine allow the development of automated vision systems that use digital image processing techniques along with machine learning methodologies to give reliable and automatic analysis of specimens in different application fields. This is the case of urinoculture, a screening test typically done on hospitalized patients and pregnant women. In fact, the urinary tract infections, together with those of the respiratory tract, are of great clinical relevance for the high frequency with which they are found in common medical practice and because of the complications arising therefrom. They are mainly caused by Gram-negative microorganisms, with a high prevalence of Escherichia coli (E.Coli, 70\%), even if clinical cases frequently occur where complicated infections are caused by Grampositive or multi-resistant germs, on which the common antimicrobial agents are

(C) Springer International Publishing Switzerland 2015

V. Murino and E. Puppo (Eds.): ICIAP 2015, Part I, LNCS 9279, pp. 635-646, 2015.

DOI: $10.1007 / 978-3-319-23231-7 \_57$ 
inevitably ineffective, leading to therapeutic failures. Actually, manual methods for urinoculture screening are work and time intensive, requiring visual inspection by a biomedical scientist for semi-quantitative scoring of each sample. This impacts on laboratory throughput and induces a poor use of qualified resources for what are predominantly negative screens. Actually, in the standard protocol, the urine sample is seeded on a Petri plate that holds a culture substrate, used to artificially recreate the environment required for the bacterial growth, and incubated at $37^{\circ} \mathrm{C}$ overnight. After the incubation, each plate is examined by a human expert.

Even if some interesting research has been carried out in recent years, obtaining an overview of the state-of-the-art in image processing solutions to the automatic analysis of Petri plates is difficult, since results are published in various domains - from food and beverage safety to environmental control and specific clinical analyses [1-3] -, based on different data sets, and often related to subtle variations of the core problem (like, f.i., in [4], where the colony classification problem is addressed with promising results, but with respect to a very small number of images and based only on the determination of isolated colonies).

The tool we propose, is able of handling the whole examination cycle starting from the automatic acquisition of the plate with a color camera and ending with the identification of the type of infection(s) and with the assessment of its severity through Support Vector Machines (SVMs). The system has been tested and evaluated on real samples provided by DIESSE Ricerche Srl, Siena, and acquired in real operative conditions.

The paper is organized as follows. Section 2 introduces the problem and describes the preprocessing procedure, from the image acquisition phase to the background removal. Section 3 presents the classification methods and shows experimental results, whereas Section 4 defines the procedure used to detect each single colony. Finally, conclusions are given in Section 5 .

\section{Automatic Image Analysis of Petri Plates}

In order to correctly recognize the infection type and to precisely estimate the bacterial load, it is fundamental to grab a good quality image of the Petri plate. To avoid imperfections due to manual plate handling, the images are captured by an automatic camera setup (Fig. 1). After the acquisition, a suitable preprocessing step is applied to locate the region of interest (the Petri plate), and to grant that it is in an appropriate position inside the field of view. At this point, the image is saved along with auxiliary information. The automatic acquisition is performed as follows: a simple and fast algorithm, based on change detection [5] and morphological filtering [6], is applied and the image is acquired only when the plate is correctly positioned, the scene is well illuminated, and no movements are observed. Before saving the image, the Petri dish is isolated from the rest of the scene using a Random Hough circle transform [7] to detect the circular Region of Interest (RoI).

The acquisition setup has been used in a real application scenario at DIESSE Ricerche premises, to collect a dataset of 253 images, subsequently divided into 


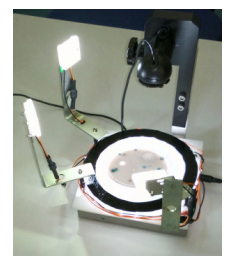

Fig. 1. The automatic acquisition set.

a training, a validation, and a test set, containing 154, 64, and 35 images, respectively. As a requirement, eight different classes of infection were detected, namely: E.Coli, KES (Klebsiella, Enterobacter, Serratia), Enterococcus Faecalis, Streptococcus Agalactiae, Pseudomonas, Proteus, Staphylococcus Aureus, and Candida.

\subsection{Selection of the Chromatic Space}

Since a chromogenic medium (Uriselect 4) is used as ground seed, the color of the pixels is the most important feature for classifying the different colonies. During preliminary studies, the background color distribution has been analyzed in four different color spaces (i.e., RGB, HSV, CIE-Lab, and YCrCb). The chromatic data relative to the background in each chromatic space have been accumulated and the Dunn's Index has been used to give a quantitative ranking (based on the Centroid Linkage distance and the Centroid Diameter dispersion [8]):

$$
\begin{aligned}
& D I(X)=\min _{1 \leq i \leq k}\left\{\min _{1 \leq i \leq k}\left\{\frac{\delta\left(X_{i}, X_{j}\right)}{\max _{1 \leq s \leq k}\left\{\Delta\left(X_{s}\right)\right\}}\right\}\right\} \\
& \delta\left(X_{i}, X_{j}\right) \triangleq \frac{1}{\left|X_{i}\right|+\left|X_{j}\right|}\left(\sum_{\vec{c} \in X_{i}} d\left(\vec{c}, C_{X_{j}}\right)+\sum_{\vec{c} \in X_{j}} d\left(\vec{c}, C_{X_{i}}\right)\right) \\
& C_{X i} \triangleq \frac{1}{\left|X_{i}\right|} \sum_{\vec{c} \in X_{i}} \vec{c} ; C_{X_{j}} \triangleq \frac{1}{\left|X_{j}\right|} \sum_{\vec{c} \in X_{j}} \vec{c} \\
& \Delta\left(X_{i}\right) \triangleq 2\left(\frac{\sum_{x \in X_{i}} d\left(x, C_{X_{j}}\right)}{\left|X_{i}\right|}\right)
\end{aligned}
$$

where $\vec{c}$ is the chromatic vector $(a, b)$ of each pixel, while the other variables have an obvious meaning. Experiments have shown that CIE-Lab has a higher ranking and also gives increased separation between the color of the eight infections and the background. CIE-Lab [9] has been selected for the chromatic description. It is known that the use of $(a, b)$ chromaticity coordinates only, makes the histogram more stable with respect to differences in illumination and local variations caused by shadows [10]. Moreover, if only the $(a, b)$ chromaticity components are used, the background colors concentrate in a very compact and stable region (Fig. 2).

\subsection{Background Removal}

The classification of infections is performed in a hierarchic way. As a preliminary step, the colonies are segregated from the background by a background-removal process based on chromatic information about the specific chromogenic medium 


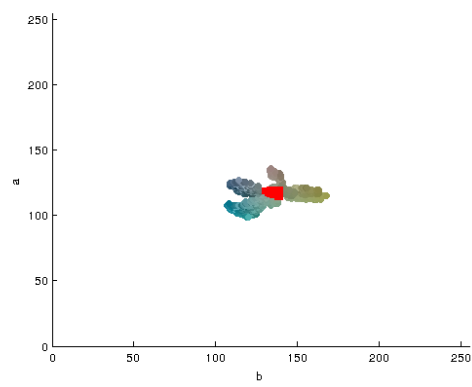

Fig. 2. Background $(a, b)$ color distribution (marked in red) compared to the distribution of the eight infections.

used in the culture (Uriselect 4, in this study). To this end a suitable chromatic background model has been defined.

Background Modeling - To obtain a chromatic description of the background, a supervised training technique is adopted, during which a human expert selects about 40 different regions belonging to the background of different images coming from the training set. The chromatic components $(a, b)$ of the pixels belonging to such regions are accumulated to represent the typical background chromatic values. The samples have been preliminarily filtered by a fast vector median filter [11] to reduce the effect of noisy samples. The color histogram distribution is estimated by a Parzen-window with a Gaussian shape [12].

Foreground Modeling - A similar approach has been used to model the foreground (infected) regions. Again a supervisor selects about 40 regions belonging to each different infection classes coming from the images of the training set. Again a Parzen-window approach is used to estimate the probability of the $(a, b)$ chromatic components for each infection class. So, finally, we are left with eight estimates of the conditional probability density function relative to the eight possible infections considered. These functions are then compared with the background probability density function to define the chromatic regions that can give rise to classification uncertainty. The uncertainty region is obtained as a union of the intersections of some binary masks obtained by imposing a threshold on the probability level of the background and of the infected regions. In formulae:

$$
\begin{aligned}
& U_{M}=\bigcup_{i=1}^{8} C_{i} \\
& C_{k}=\bigcap\left(B_{\tau}, I_{\tau, k}\right) ; k \in[1,8] \\
& B_{\tau}=\left\{\begin{array}{lll}
1 & \text { if } \vec{c}_{b a c k} \in \text { background \&\& } p\left(\vec{c}_{b a c k}\right) \geq \tau \\
0 & \text { otherwise }
\end{array}\right. \\
& I_{\tau, j}=\left\{\begin{array}{lll}
1 & \text { if } & \vec{c}_{j} \in j \text {-region \&\& } p\left(\vec{c}_{j}\right) \geq \tau \\
0 & \text { otherwise }
\end{array}\right.
\end{aligned}
$$




$$
\begin{aligned}
& \tau=\min \left\{t_{1}, t_{2}\right\} \\
& t_{1}=\min _{k}\left\{\iint_{p\left(\vec{c}_{j}\right) \geq k} p\left(\vec{c}_{j}\right) d \vec{c} \geq \alpha \mid \vec{c}_{j} \in I_{\tau, j} ; j=1, \ldots 8\right\} \\
& t_{2}=\underset{p\left(\vec{c}_{\text {back }}\right) \geq t_{2}}{\iint_{t_{\text {a }}} p\left(\vec{c}_{\text {back }}\right) d \vec{c} \geq \alpha ; \vec{c}_{\text {back }} \in \text { background }}
\end{aligned}
$$

where $\tau$ is a constant threshold imposed to be sure that a percentage of at least $\alpha$ (say $\alpha=90 \%$ ) of the background pixels belong to each set, $\vec{c}_{j}$ is the chromatic vector of a pixel belonging to the $j$-th infection type, and $\vec{c}_{b a c k}$ is a pixel belonging to the background.

At this point the mask relative to each infected region $\left(C_{k}\right.$ for the $k$-th infected region) is intersected with the background mask to give a superposition binary mask. Finally the union $U_{M}$ of the whole set of superposition masks is computed. It is easy to see that the final mask comprises all the chromatic coordinates that belong with a certain degree of probability (actually more than $\tau)$ both to the background and to one of the infected regions. The chromatic coordinates of the points belonging to the final mask are marked as uncertain and used during the subsequent classification stage.

Background Segmentation - Even if the background chromatic model is quite stable and accurate, some minor chromatic variations are still present. To accommodate for these variations, a Mean-Shift segmentation [13] algorithm is used to associate each image pixel to the corresponding modal density value in the $(a, b)$ space (actually, as usual, the chromatic coordinates are mixed with the positional coordinates of each pixel so the Mean-Shift algorithm runs in a four-dimensional space). The modal value so obtained, is compared with the background chromatic model (by using the corresponding likelihood), to establish if the pixel belongs to the background region or not. In this way a first approximated background segment is obtained. Unfortunately such an approximation is rarely satisfying because the background slightly changes its colors according to the type of bacterium grown on the plate. To solve this problem, we use the previously defined uncertainty chromatic mask $U_{M}$. In particular, when some pixels belong to the mask (i.e., they cannot be assigned with reasonable certainty on the basis of chromatic coordinates), we assign them to a fictitious class of uncertain points. In the image space such points form a set of uncertainty regions and specific post-processing steps are applied to each of them.

Uncertainty regions are analyzed taking into account also local spatial properties like discontinuities (edges), that are typically present between the colonies and the background. In particular, we have considered the following five classes:

(1) Colonies (E. Coli, KES, E. Faecalis, S. Agalactiae);

(2) Background without edges (blue in Fig. 3);

(3) Background with edges (pink in Fig. 3);

(4) Uncertainty region without edges (red in Fig. 3);

(5) Uncertainty region with edges (green in Fig. 3). 


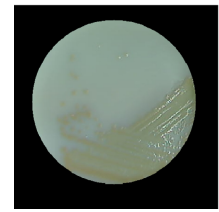

(a)

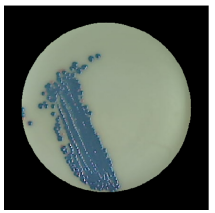

(d)

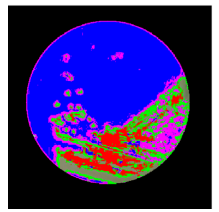

(b)

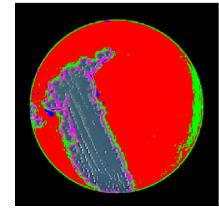

(e)

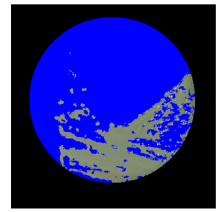

(c)

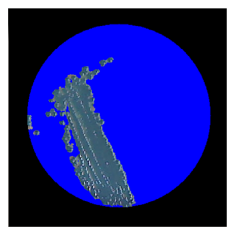

(f)

Fig. 3. Images (a) and (d) represent two dishes in which Pseudomonas and KES colonies are present; images (b) and (e) show the five different regions present on each Petri dish, and (c) and (f) the results obtained after the application of the described background segmentation procedure.

The classification procedure is the following: at first the background chromatic model is used to decide if a segment (obtained by Mean-Shift segmentation) belongs to a colony, to the background or to an uncertainty region. Thereafter, a Sobel [14] based edge enhancement is applied to distinguish class (3) and (5). Then, uncertainty regions (4) and (5) are analyzed to assign them to the background or to the colonies. During the experiments, it has been noted that if the HSV space [15] is used to describe uncertainty regions then, if the region belongs to the background, the $\mathrm{H}$ (hue) channel shows a peak that is near to the one obtained by taking the same histogram but relative to the region of class (2). To take advantage of this observation, the uncertainty regions of class (4) and (5) are analyzed separately by applying the same procedure, summarized in the following.

1. The Otsu method [16] is used to threshold the $\mathrm{H}$ values of uncertainty regions in class (4) and (5) and to segment them;

(a) Based on the computed threshold, the considered uncertainty region is divided into two sub-regions;

(b) The histograms of the two sub-regions are computed and compared, to establish if a significant separation exists (i.e. if the peaks of the two sub-regions are far enough in the histogram of the $\mathrm{H}$ channel). If the peaks are separated, the two sub-regions are identified as (6) and (7), respectively, and processed separately. If the peaks are not separated, the two sub-regions are kept together;

2. The peaks of the histogram ( $\mathrm{H}$ channel) of the region of class (2) and of the analyzed regions $((6)$ and $(7)$ separately or together, depending on the previous step) are compared; 
3. If the peaks of the background and of the current region ((6) and (7) separately or together) are distant enough, the current region cannot be assigned to the background and is passed to the final classification step to assign it to the appropriate colony;

4. Instead, if the peaks are almost coincident, the uncertainty region shows a $\mathrm{H}$ value similar to the background, and it is assigned to it.

In other words, for both regions (4) and (5), we identify different sub-regions (if any) and, for each "homogeneous" zone, we establish its membership to the background or not.

Further problems arise when considering Candida colonies, since their color is practically the same as the culture ground. To classify this infection, an ad hoc procedure has been devised, particularly focused on analyzing segments belonging to region (3).

The performance of the background segmentation method has been evaluated on the test set comprising 33 images. The results are reported in Table 1.

Table 1. Results of the background extraction preprocessing.

\begin{tabular}{|l|c|}
\hline Background extraction & \\
\hline Total Number of background pixels & 1091413 \\
Background pixels correctly classified & 1068445 \\
Background pixels incorrectly classified & 22968 \\
Accuracy & $97,9 \%$ \\
\hline
\end{tabular}

\section{Classification}

After the background removal process has been performed, the residual regions, not belonging to it, are classified. A multi-stage classification approach is used for this aim.

The classification of the bacteria grown on a Petri plate is a very difficult problem and it is usually carried out by a qualified biologist. Human experts can use their visual skills and a priori knowledge to solve the task. Due to the use of a chromogenic ground seed, the most important feature for recognizing different type of bacteria is color. In fact, Uriselect 4 is specifically designed to support the infections classification through the hue taken by the colonies. This is due to different bacterial enzymes whose activity produces a peculiar color:

- $\beta$-galactosidase and tryptophanase: red-pink, typical for E. Coli infections;

- $\beta$-glucosidase: turquoise blue, typical for Enterococcus Faecalis infections; the simultaneous presence of $\beta$-galactosidase turns the color to purplish blue, typical for the KES group infections;

- tryptophane désaminase: yellow/orange, typical for Proteus-ProvidenciaMorganella infections. 
Therefore, the first step in our multi-stage classification algorithm is to recognize the previous three classes by using chromatic features. This lead to a pre-classification which can be further used to recognize subclasses among the main classes. The pre-classification step divides the infections into three main chromatic groups: red (E. Coli), blue (Enterococcus Faecalis, KES and Streptococcus Agalactiae), and yellow/orange (Pseudomonas, Proteus and Staphylococcus Aureus). Such a division is obtained by an SVM (Support Vector Machine) classifier with a Gaussian kernel, with $\gamma=0.1$ and $C=7.75$ (the parameters have been chosen via a trial-and-error procedure). SVMs has been implemented by using the Weka software tools (http://www.cs.waikato.ac.nz/ml/weka/). To collect the feature vectors for the classifier, we have first executed a Mean-Shift segmentation algorithm and then, for each segment, the $(a, b)$ color components in the CIE-Lab space have been extracted. The results of this pre-classification step are reported in Table 2.

Table 2. Classification results for the three main colors based on an SVM classifier.

(a) Accuracy

\begin{tabular}{|l|c|c|}
\hline \multicolumn{3}{|c|}{ SVM classifier for the three main colors } \\
\hline Total Number of Segments & 13292 & Percentage \\
\hline Incorrectly Classified Segments & 36 & $0.2708 \%$ \\
\hline Correctly Classified Segments & $\mathbf{1 3 2 5 6}$ & $\mathbf{9 9 . 7 2 9 2 \%}$ \\
\hline
\end{tabular}

(b) Confusion Matrix

\begin{tabular}{|c|c|c|}
\hline \multicolumn{3}{|c|}{ Red Blue Yellow/Orange } \\
\hline 4479 & 0 & 10 \\
\hline 0 & 8691 & 12 \\
\hline 14 & 0 & 86 \\
\hline
\end{tabular}

Only E. Coli produces red colonies among the eight diverse types of infections considered, so it can be recognized in the first step. Instead, for the other classes, some more information is needed to recognize each specific kind of infection.

For the blue class (E. Faecalis, KES, St. Agalactiae), the first important issue is the presence of residual background segments not correctly identified by the background segmentation module. Even if the module is quite efficient, experimentally, it turned out that even a small amount of background badly affects the blue class classification performance. The background extractor output and the pre-tag have been used as an input for a GrabCut algorithm [17], that allows to remove the majority of the remaining background segments. Thereafter, an SVM classifier with a Gaussian kernel, with $\gamma=0.12$ and $C=1$ (the parameters have been chosen via a trial-and-error procedure) has been used. Results for the classification of blue infections are reported in Table 3.

Furthermore we find out unpredictable chromatic variations produced when different species of bacteria overlap on the same Petri dish. This is the case of the contemporary presence of E. Coli and E. Faecalis, that produces an overlapping region which looks like KES. Therefore, we use this a priori information to predict which colonies are probably present also in the overlapping regions, starting from the classification of segregated colonies. The colony dimension is a useful feature to discriminate Enterococcus Faecalis (small colonies, 0,5 - 1,5 $\mathrm{mm}$ for the diameter) from KES $(2-3 \mathrm{~mm})$. Taking advantage from the procedure capable to recognize isolated colonies (Section 4) we then use the dimension of a single 
colony as a feature to improve the classification performance. Based on both the color component $(a, b)$ and the dimension of the colonies, we train another SVM classifier with a polynomial kernel, with degree $=3, \gamma=0.25$, and $C=1$. Results for this classification are reported in Table 4.

A similar procedure was also implemented with respect to the yellow class (Pseudomonas, Proteus and Staphylococcus Aureus), obtaining very promising results. Anyway, such results are very preliminary, since they were achieved on a too small set of images to be actually statistically significant.

Table 3. Classification results for the blue class.

(a) Accuracy

\begin{tabular}{|l|c|c|}
\hline \multicolumn{3}{|c|}{$\begin{array}{c}\text { SVM classifier for KES, Faecalis } \\
\text { and Agalactiae }\end{array}$} \\
\hline Total Number of Segments & 7122 & Percentage \\
\hline Incorrectly Classified Segments & 1240 & $17.4108 \%$ \\
\hline Correctly Classified Segments & $\mathbf{5 8 8 2}$ & $\mathbf{8 2 . 5 8 9 2} \%$ \\
\hline
\end{tabular}

(b) Confusion Matrix

\begin{tabular}{|ccc|}
\hline KES & Faecalis & Agalactiae \\
\hline 3021 & 191 & 12 \\
604 & 2548 & 213 \\
17 & 203 & 313 \\
\hline
\end{tabular}

Table 4. Classification results for the blue class using both color and colony dimension as input features.

(a) Accuracy

\begin{tabular}{|l|c|c|}
\hline \multicolumn{3}{|c|}{$\begin{array}{c}\text { SVM classifier for KES, Faecalis } \\
\text { and Agalactiae isolated colony }\end{array}$} \\
\hline Total Number of Colonies & 226 & Percentage \\
\hline Incorrectly Classified Colonies & 30 & $13.2743 \%$ \\
\hline Correctly Classified Colonies & $\mathbf{1 9 6}$ & $\mathbf{8 6 . 7 2 5 7} \%$ \\
\hline
\end{tabular}

(b) Confusion Matrix

\begin{tabular}{|c|c|c|}
\hline KES & Faecalis & Agalactiae \\
\hline 76 & 0 & 0 \\
\hline 24 & 91 & 1 \\
\hline 0 & 5 & 29 \\
\hline
\end{tabular}

Finally a distinct procedure has been developed to detect the Candida infections. This is a very difficult task because Candida looks semi-transparent, with a color which is nearly the same as the culture ground. In fact, biologists, in order to find out the presence of Candida, usually rotate and move the plate in different positions, an operation aimed at finding any additional useful feature (reflections, thickness of the colony, etc.). Since the camera catches just a frontal view of the Petri dish, a 2D image does not allow such a possibility. Therefore, Candida was revealed by detecting eventual edges inside the background (caused by the colony extrusions originating small reflections). Unfortunately, an edge can be also due to noise; therefore, in order to reduce the number of false positives, we used the shape of the colony, generally circular, as a fundamental feature to distinguish colonies from noise. Single non overlapping colonies were then searched on the edge mask and, based on their number, we were finally able to establish the presence of Candida or not (see Fig. 4). 


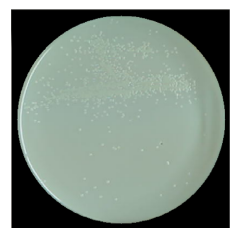

(a)

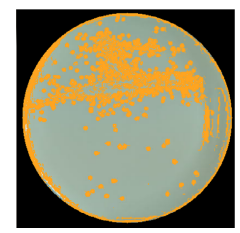

(b)

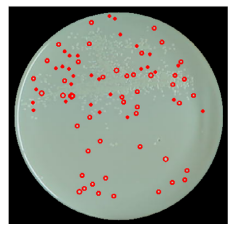

(c)

Fig. 4. In (a), the original image; in (b) the identified edges within the background, in orange; in (c), Candida colonies found on the Petri dish.

\section{Colony Detection and Bacterial Count}

In order to perform the bacterial count, a multistage algorithm has been developed that, at first, searches only for single, not overlapping colonies, and then tries to enucleate colonies belonging to slightly overlapping regions. A binary image is first constructed, in which the background is represented by the ground seed and the foreground by the colonies. Single colonies show a roughly circular shape and can be easily identified based on this feature. In particular, for each connected component in the binary image, we calculate the least enclosing circle and then, if the ratio between the circular area and the area of the given connected component is under a fixed threshold (chosen via a trial-and-error procedure), such a component is supposed to be a colony. Obviously, this simple approach is not effective in massive overlapping regions, where a different procedure has to be used. Therefore the convexity of each colony contour is calculated and sub-contours with convex shape are found; then, the best ellipse (in the least square sense), that fits each sub-contour, is selected. A score matrix, that takes into account both the axes rate and the ellipses' points belonging (or not) to the contour, is constructed and used to remove non-relevant ellipses (Fig. 5).

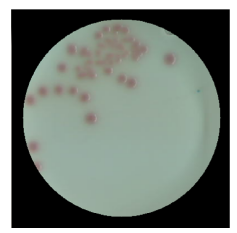

(a)

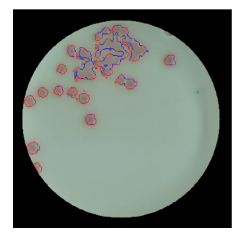

(b)

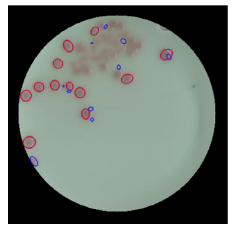

(c)

Fig. 5. In (a) the original image; in (b) contours are evidenced (blue: the concave part; red: the convex part); in (c) the ellipses, with a high score (red), and a low score (blue).

Using the dimension of the discovered single colonies, combined with the area of the infected region, our system calculates the infection severity (or the bacterial load), which is an estimate of the number of microorganisms per milliliter of urine (UFC/ml), expressed by using a logarithmic evaluation scale. The actual measurement value is obtained by multiplying the number of bacterial colonies counted on the dish by the inverse of the seeding dilution rate. Based on this 
procedure, we obtain an accuracy of $92,1 \%$ (our estimations agree with those of the biologists for 233 out of 253 images constituting the dataset).

\section{Conclusions}

In this paper, an automatic procedure to detect and classify urinary infections was described, able to obtain a good accuracy on the most common bacteria present in humans. Preliminary results were presented. It is a future matter of research to refine the classification procedure related to colonies with very similar colors and also revealing the presence of Candida. Finally, the tool will be also extended to treat diverse types of culture grounds, possibly transparent and based on the action of different enzymes (so producing different chromatic reactions).

\section{References}

1. Ogawa, H., Nasu, S., Takeshige, M., Funabashi, H., Saito, M., Matsuoka, H.: Noise-free accurate count of microbial colonies by time-lapse shadow image analysis. Journal of Microbiological Methods 91(3), 420-428 (2012)

2. Clarke, M.L., Burton, R.L., Hill, A.N., Litorja, M., Nahm, M.H., Hwang, J.: Low-cost, high-throughput, automated counting of bacterial colonies. Cytometry Part A 77(8), 790-797 (2010)

3. Brugger, S.D., Baumberger, C., Jost, M., Jenni, W., Brugger, U., Mühlemann, K.: Automated counting of bacterial colony forming units on agar plates. PLoS ONE $\mathbf{7}(3)$, e33695 (2012)

4. Ferrari, A., Signoroni, A.: Multistage classification for bacterial colonies recognition on solid agar images. In: Proceeding of IEEE IST 2014, pp. 101-106 (2014)

5. Ilsever, M., Unsalan, C.: Two-Dimensional Change Detection Methods: Remote Sensing Applications, pp. 7-21. SpringerBriefs in Computer, Science (2012)

6. Maragos, P., Pessoa, L.: Morphological filtering for image enhancement and detection. In: Handbook of Image and Video Processing. Academic Press (2005)

7. Mount, D., Netanyahu, N.: Efficient Randomized Algorithms for Robust Estimation of Circular Arcs and Aligned Ellipses. Computational Geometry 19(1), 1-33 (2001)

8. Halkidi, M., Batistakis, Y., Vazirgiannis, M.: On Clustering Validation Techniques. Journal of Intelligent Information Systems 17, 107-145 (2001)

9. Hunter, R.S.: Photoelectric color difference meter. In: Proceedings of the Winter Meeting of the Optical Society of America, vol. 38(7), p. 661. JOSA (1948)

10. Saxe, D., Foulds, R.: Toward robust skin identification in video images. In: 2nd Int. Face and Gesture Recognition Conf. (1996)

11. Smolka, B., Szczepanski, M., Plataniotis, K.N., Venetsanopoulos, A.N.: Fast modified vector median filter. In: Skarbek, W. (ed.) CAIP 2001. LNCS, vol. 2124, p. 570. Springer, Heidelberg (2001)

12. Heidenreich, N., Schindler, A., Sperlich, S.: Bandwidth selection for kernel density estimation: a review of fully automatic selectors. Adv. Stat. Anal. 97, 403-433 (2013)

13. Comaniciu, D., Meer, P.: Mean-shift: A robust approach toward feature space analysis. IEEE Trans. Pattern Anal. Machine Intell. 24, 603-619 (2002) 
14. Gonzalez, R., Woods, R.: Digital Image Processing, pp. 414-428. Addison Wesley (1992)

15. Smith, A.R.: Color gamut transform pairs. In: SIGGRAPH 1978 - Proceedings of the 5th Annual Conference on Computer Graphics and Interactive Techniques, vol. 12(3), pp. 12-19 (1978)

16. Otsu, N.: A threshold selection method from gray-level histograms. IEEE Trans. on System, Man and Cybernetics 9, 62-66 (1979)

17. Rother, C., Kolmogorov, V., Blake, A.: GrabCut: Interactive foreground extraction using iterated graph cuts. ACM Trans. Graph. 23, 309-314 (2004) 